\title{
Physicochemical characterization of pulp and nanofibers from kenaf stem.
}

\begin{abstract}
The aim of this study was to isolate cellulose nanofibers from kenaf (Hibiscus cannabinus) stem using chemo-mechanical treatments. The fiber purification method included pulping and bleaching processes whereas the mechanical treatments employed to isolate kenaf nanofibers were grinding and high pressure homogenizing. Kenaf nanofibers were found to have diameters in the range of $15-80 \mathrm{~nm}$ while most nanofibers have diameters within the range $15-25 \mathrm{~nm}$. Fourier transform infrared spectroscopy (FTIR) showed that the chemical treatments removed lignin and most of the hemicelluloses from the fibers. The thermal characteristics of the fibers were analyzed using the technique of thermogravimetric analysis (TGA) which demonstrated that these characteristics were enhanced noticeably both for the bleached pulp and nanofibers. On the other hand, the X-ray analysis indicated that both chemical and mechanical treatments can improve the crystallinity of fibers.
\end{abstract}

Keyword: Kenaf Stem; Nanofiber; Crystal structure; Thermal properties; Electron microscopy. 